\title{
The Origin and Differentiation of Planet Mercury
}

\author{
Bernard Charlier ${ }^{1}$ and Olivier Namur ${ }^{2}$
}

1811-5209/19/0015-0009\$2.50 DOI: 10.2138/gselements.15.1.9

$\mathbf{U}$ nique physical and chemical characteristics of Mercury have been revealed by measurements from NASA's MESSENGER spacecraft. The closest planet to our Sun is made up of a large metallic core that is partially liquid, a thin mantle thought to be formed by solidification of a silicate magma ocean, and a relatively thick secondary crust produced by partial melting of the mantle followed by volcanic eruptions. However, the origin of the large metal/silicate ratio of the bulk planet and the conditions of accretion remain elusive. Metal enrichment may originate from primordial processes in the solar nebula or from a giant impact that stripped most of the silicate portion of a larger planet leaving Mercury as we know it today.

KEYWords: MESSENGER, BepiColombo, terrestrial planet, magma crystallization, interior structure

\section{INTRODUCTION}

Mercury is the closest planet to the Sun and an end-member terrestrial body in terms of internal structure and surface composition in our solar system. As described by Solomon and Byrne (2019 this issue), Mercury has been explored both by Earth-based telescopes and by two NASA spacecraft missions: Mariner 10 (1974-1975) and MESSENGER (2008-2015). The third mission to Mercury, BepiColombo, was launched by the European Space Agency (ESA) and the Japanese Space Agency (JAXA) on 20 October 2018 and will reach the orbit of Mercury in 2025. In this article, we review the major characteristics of the interior structure and surface of Mercury, focusing on the size and composition of the different layers of this planet which had an early history dominated by differentiation through mantlecore segregation followed by magma-ocean solidification, volcanic activity, cratering and impact melting. All these processes led to the present-day structure of Mercury as depicted in Figure 1. The most striking characteristic of Mercury, besides its small size, is the relatively large mass fraction that the core represents $(\sim 65 \%$ of the total mass of the planet). Mercury also has a thick crust $(\sim 35 \mathrm{~km})$ relative to the size of the mantle $(\sim 400 \mathrm{~km})$ and, possibly, an FeS layer at the core-mantle boundary. The inner core is solid, the outer core is molten. The composition of the surface of Mercury (Nittler and Weider 2019 this issue) is also unique, with very low iron but high sulfur contents, which point to highly reducing conditions during planetary differentiation.

1 Department of Geology, University of Liege
4000 Sart Tilman, Belgium 4000 Sart Tilman, Belgium

2 Department of Earth and Environmental Sciences, KU Leuven 3001 Leuven, Belgium

E-mail: olivier.namur@kuleuven.be
A major unsolved question for Mercury is the origin of its much higher metal/silicate fraction (i.e., metal enrichment) compared to other terrestrial planets. Several processes have been invoked to explain this metal enrichment (Ebel and Stewart 2018). It may be related to the composition of the inner part of the nebula where Mercury formed, but other mechanical processes may also have occurred after the formation of the (proto-)planet. We present some possible causes of metal/ silicate fractionation, including element fractionation in the solar nebula (condensation sequence, dynamical mechanism), impact erosion, and high-temperature evaporation of the silicate shell. Mercury's formation processes and internal structure are now being revealed thanks to the data collected by MESSENGER, but much remains to be explained. The BepiColumbo mission will undoubtedly answer more questions.

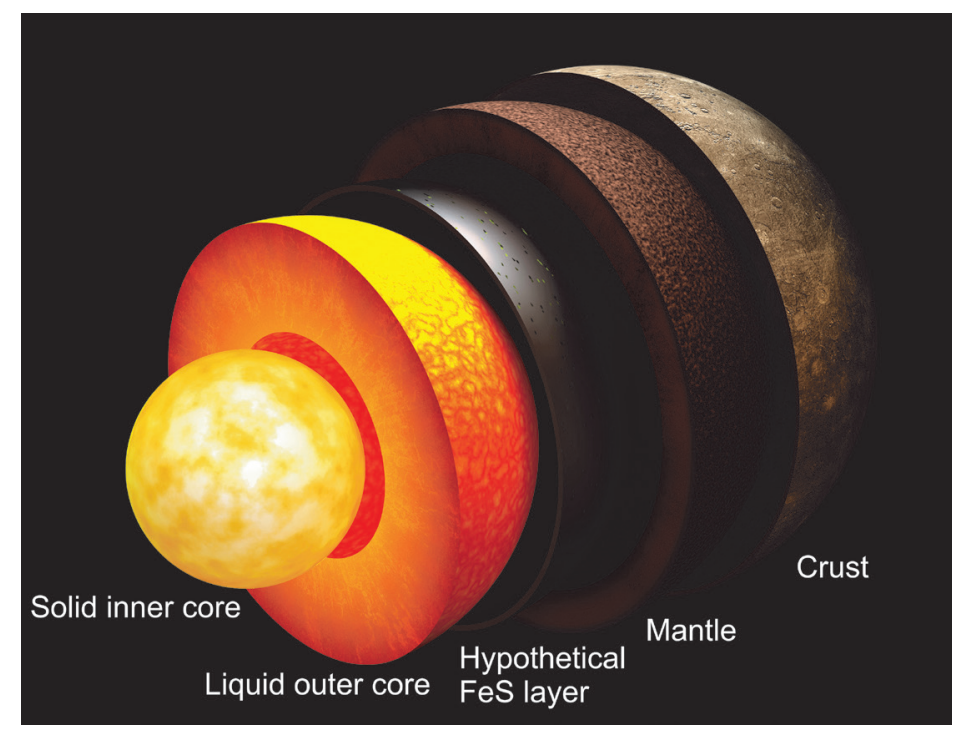

FigurE 1 The interior structure of Mercury. The relatively thick crust $(\sim 40 \mathrm{~km})$ overlies the comparatively thin mantle $(\sim 400 \mathrm{~km})$. A hypothetical iron sulfide layer might occur at the core-mantle boundary, overlying the liquid outer core (radius of $\sim 2,000 \mathrm{~km}$ ). The innermost part of Mercury is probably a solid core having a radius of $<1,000 \mathrm{~km}$. Figure Copyricht @ Mark A. Garlick. 


\section{THE DIFFERENTIATION OF MERCURY}

The internal structure of Mercury has evolved over time in conjunction with the cooling of the planet. A primordial fully molten state (FIG. 2A) enabled the separation of a metallic core from a silicate magma ocean. Upon cooling, these two chemically distinct reservoirs started crystallizing, forming a growing solid inner core and a silicate mantle, potentially capped by a graphite primary crust that floated on the silicate mantle (Vander Kaaden and McCubbin 2015) (FIG. 2B). Inner-core crystallization and latent-heat release led to an initial phase of mantle heating that produced strong mantle convection and partial melting of the mantle by decompression. Magmas produced in the mantle moved to the surface where lava flow accumulation formed the secondary volcanic crust (FIG. 2C). Progressive cooling of the mantle below its solidus temperature, as well as planetary contraction, both led to the termination of significant magmatism at about $3.6 \mathrm{Ga}$ (FIG. 2D). Further morphological modification of the crust is related to meteorite bombardment, which produced cratering and impact melting. A peculiar feature of Mercury is the very low abundance of iron, and especially $\mathrm{Fe}^{2+}$, at the surface, despite the bulk planet being significantly enriched in iron compared to other terrestrial planets and meteorites. This indicates that highly reducing conditions (low-oxygen abundances) prevailed during differentiation and that nearly all of the iron was extracted to the core in its metallic form.

\section{The Crust}

Surface imaging and compositional data acquired by MESSENGER have revealed that the physical and compositional characteristics of Mercury's present-day surface are largely controlled by volcanism and impacts (see Thomas and Rothery 2019 this issue). The oldest terrains are dated to $4.1 \mathrm{Ga}$ using crater density, and the youngest to $\sim 3.5 \mathrm{Ga}$ (Marchi et al. 2013). The surface of Mercury is covered by a range of volcanic features. The thickness of the crust is estimated to be $35 \pm 18 \mathrm{~km}$ using the geoid-to-topography ratio (Padovan et al. 2015).

The primordial crust of Mercury might have been a graphite flotation lid, which is probably the source of the carbon that is identified today at the surface of the crust (an average $\mathrm{C}$ concentration of $\sim 1 \mathrm{wt} \%$ ) and mostly concentrated in low-reflectance material (up to $4-5 \mathrm{wt} \% \mathrm{C}$ ) (Peplowski et al. 2016). Under reducing conditions, $\mathrm{C}$ did not partition strongly into the core, but, rather, stayed in Mercury's silicate magma ocean (Li et al. 2017). Magmaocean solidification together with low $\mathrm{C}$ solubility in silicate melts might, therefore, have led to early saturation in solid graphite and its flotation on a residual melt from the magma ocean (Vander Kaaden and McCubbin 2015).

Although carbon at the surface may be a remnant from the primary crust, the bulk of the crust as we see it today was formed during secondary volcanic processes. Different terranes can be distinguished based on their composition (Nittler and Weider 2019 this issue) and on their mineralogical characteristics (Namur and Charlier 2017). The high-Mg province (likely dominated by olivine)

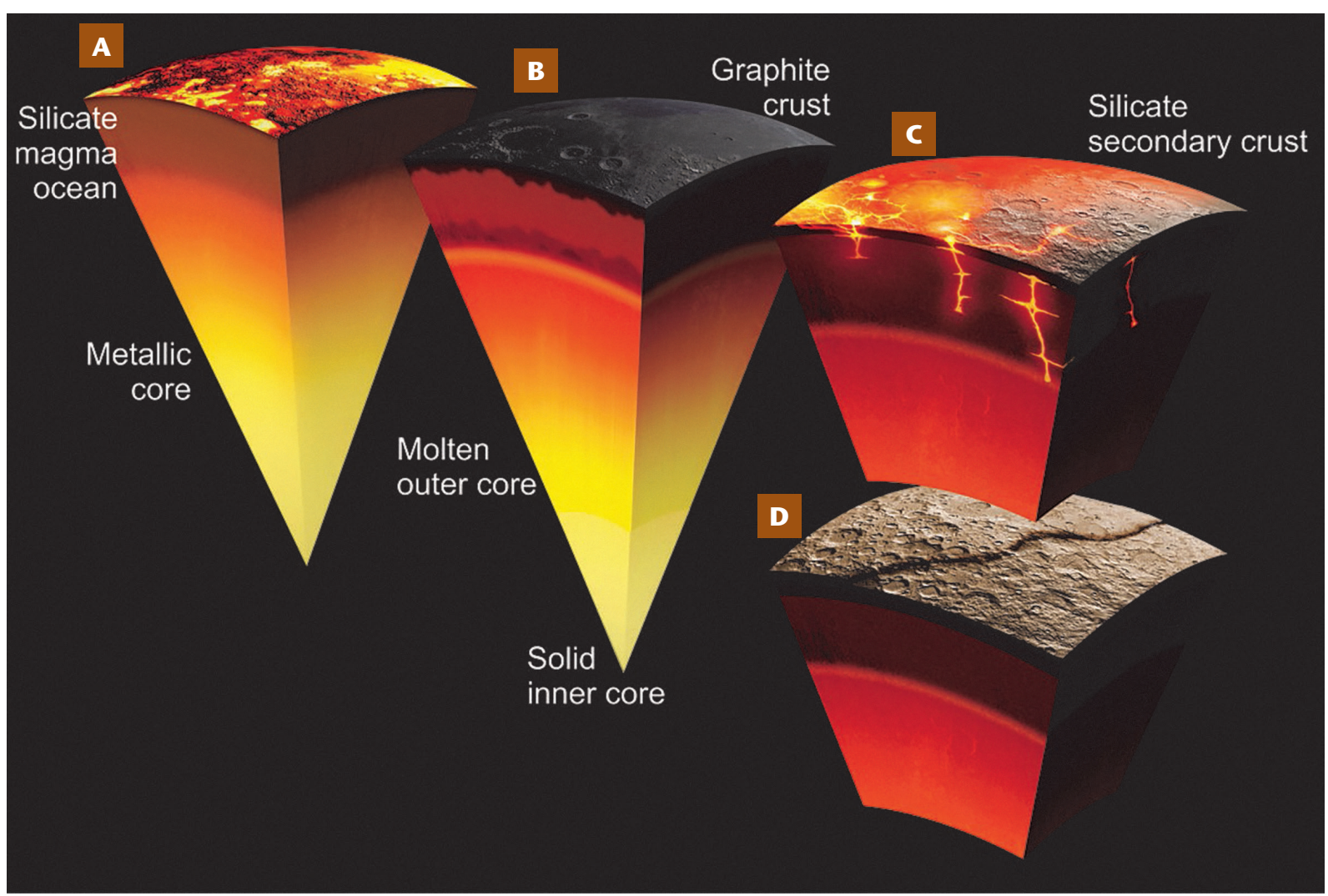

Figure 2 The four key events in the origin hypothesis of Mercury. (A) Global melting of the planet and separation of a metallic core from a silicate magma ocean. (B) Cooling of the planet, progressive crystallization of the solid inner core, potential formation of an FeS layer at the core-mantle boundary, and crystallization of the magma ocean to produce the primordial mantle and a graphite flotation crust. (C) Partial melting of the mantle, production of the secondary crust, and synchronous cratering of the surface. (D) Termination of major volcanism at about $3.6 \mathrm{Ga}$ and global planetary contraction. Figure CopYright (C) Mark A. GarLick. 
contrasts with the Mg-poor and Al-rich northern volcanic plains, which are dominated by plagioclase. The surface of Mercury is also enriched in sulfur compared to other terrestrial planets and may also contain other important volatile elements such as F and Cl (Nittler and Weider 2019 this issue). Sulfur concentrations range from 0.5 to $3.5 \mathrm{wt} \%$ $\mathrm{S}$, most probably as the solid phases of $\mathrm{FeS}$ and $(\mathrm{Ca}, \mathrm{Mg}, \mathrm{Fe})$ $\mathrm{S}$ or as quenched immiscible droplets.

The late-stage evolution of Mercury's crust is marked by contraction tectonics, responsible for the formation of lobate scarps (Byrne et al. 2014), and by impact cratering. Late volcanic expressions, such as vents and pyroclastic deposits, occur most commonly associated with craters, as do bright deposits called "hollows" which are possibly related to the loss of volatiles (Blewett et al. 2011). Some late-stage processes, such as bombardment or thermal migration of elements, might also have affected the composition of the crust. Exogeneous processes that include elemental delivery from projectiles is likely and may also have contributed to the presence of carbon, which acts as a darkening agent. The proximity of Mercury to the Sun and its 3:2 spin-orbit resonance results in long days and nights with very large temperature contrasts. This in itself might affect the redistribution of some volatile elements, such as sodium, from the hot equator to the much colder poles (Peplowski et al. 2014). Due to limited MESSENGER data for alkali elements in the southern hemisphere, volatile redistribution has currently only been investigated for the northern part of the planet. New measurements by BepiColombo in the southern hemisphere will be highly informative regarding the primary or secondary origin of high-sodium abundances in the region of Mercury's northern volcanic plains.

\section{The Mantle}

Cooling and crystallization of the magma ocean were responsible for the formation of the mantle, which today has an estimated thickness of $420 \pm 30 \mathrm{~km}$ (Hauck et al. 2013) (FIG. 1). Crystallization of the magma ocean may have formed different lithologies that later partially melted to form the secondary volcanic crust. Based on high-temperature-high-pressure experiments, as well as thermodynamic modelling, primordial mantle sources were constrained to be lherzolitic and only differ by their content of clinopyroxene and a Na-bearing phase (albitic plagioclase or jadeitic pyroxene) (Namur et al. 2016b). Melt extraction and the formation of a volcanic crust progressively changed the mantle mineralogy, leaving behind the residual minerals olivine and orthopyroxene (harzburgite) (Namur et al. 2016b). Modelling also indicates that the mantle potential temperature and depth for the initial melting required for the older high-Mg region was $\sim 1,650^{\circ} \mathrm{C}$ and $360 \mathrm{~km}$, whereas for the younger lavas of the northern volcanic plains the conditions were $\sim 1,410^{\circ} \mathrm{C}$ and $160 \mathrm{~km}$. This supports a strong secular cooling of Mercury's mantle between $\sim 4.1 \mathrm{Ga}$ and $3.6 \mathrm{Ga}$. The thermal and convective states of the mantle may also have been perturbed by large impacts, which may have affected the source depth and volume of magmatism with, for example, the production of large volumes of magma in the largest basins, such as Caloris and Rembrandt.

Sulfur is also an important component of Mercury's mantle. Because sulfur behaves as a lithophile element under reducing conditions (see Cartier and Wood 2019 this issue), the solubility of $\mathrm{S}$ in the silicate melt of the magma ocean was very high, with a probable concentration ranging from 7 to $11 \mathrm{wt} \% \mathrm{~S}$ (Namur et al. 2016a). Due to low concentrations of $\mathrm{Fe}^{2+}$ in the silicate shell, it is likely that crystallization of the magma ocean produced $\mathrm{CaS}$ and MgS minerals, i.e., oldhamite and niningerite.

\section{The Core}

The mean density of Mercury is $5.43 \pm 0.01 \mathrm{~g} \cdot \mathrm{cm}^{-3}$, which indicates the presence of a large metallic core (FIG. 1). The outer liquid-core boundary is estimated to be at a radius of $\sim 2,000 \mathrm{~km}$ (Hauck et al. 2013). Thermodynamic and thermal-evolution models favor the presence of a solid inner core with a radius in the range of 1,000 to $1,500 \mathrm{~km}$ (Dumberry and Rivoldini 2015; Knibbe and van Westrenen 2018).

Experiments on metal-silicate equilibration under reducing conditions have revealed that significant $\mathrm{Si}$ can be incorporated into metal (Malavergne et al. 2010; Chabot et al. 2014), and the core of Mercury may, therefore, be mainly composed of metallic Fe and Si. Due to the relatively small size of Mercury and the large immiscibility field in the Fe$\mathrm{Si}-\mathrm{S}$ system, the $\mathrm{S}$ concentration in the Fe-Si core is probably below $2 \mathrm{wt} \% \mathrm{~S}$ (Namur et al. 2016a). The presence of an FeS layer at the mantle-core interface has been suggested by modelling of the moment of inertia of Mercury (Hauck et al. 2013). Experiments on sulfur solubility in silicate and metal melts also support the idea that an FeS layer may exist. The thickness of the FeS layer is strictly correlated to the $\mathrm{S}$ concentration in the bulk Mercury and to the conditions of oxygen fugacity, which strongly control S solubility in metal and silicate melts. For a realistic assumption of 3-5 $\mathrm{wt} \% \mathrm{~S}$ in the bulk planet and differentiation at IW-5.4 \pm 0.4 (with IW being the iron-wüstite oxygen fugacity buffer), the thickness of an FeS layer would range from $0 \mathrm{~km}$ to $90 \mathrm{~km}$. In the scenario of a primordial FeS layer, its formation would impact the composition of the silicate portion and, especially, the distribution of chalcophile elements which would then be depleted in the mantle and the crust. Even for lower bulk concentrations of sulfur in Mercury, a sulfide layer could form during crystallization of the inner core. Solid Fe-Si alloy crystals would then form the inner core, and the sulfur would be progressively enriched in the residual metal liquid, potentially reaching the sulfur capacity of the metallic liquid. Light FeS would be produced by exsolution and then float to the core-mantle boundary. In the scenario of a late-stage growing FeS layer exsolved from the outer core, no depletion in chalcophile elements would be expected in the overlying silicate magmas.

\section{FORMATION MODELS}

The debate on the origin of Mercury is intimately linked to the question of metal/silicate fractionation in the early solar nebula and in planets, as well as to the origin of metal-rich chondrites. Enstatite chondrites (EH chondrites) or, potentially, carbonaceous bencubbinite chondrites (CB chondrites) may represent the building blocks that accreted to form Mercury (Malavergne et al. 2010). These chondrites might not exactly represent the building blocks but may have sampled the Mercury feeding zone in a metal-enriched inner disk. This would imply that a common process was responsible for the principal characteristics of both the $\mathrm{EH}$ and CB chondrites as well as the characteristics of Mercury, i.e., reduced mineral assemblages, high metal/silicate ratios and minor depletion in volatiles. Processes likely to explain the origin of Mercury, as we see it today, could be classified into two end-members. One end-member is related to the evolution of the solar nebula and its ability to fractionate metal from silicate, while the other considers an increase in metal/silicate ratio through violent collisions or hightemperature evaporation on a protoplanet with an initially more modest metal fraction. 


\section{Metal-Silicate Fractionation in the Nebula}

There are several pre-accretion processes able to fractionate metal from silicate in the solar nebula (Fig. 3). These processes are based either on the removal of silicate or on the accumulation of metal, either of which will result in a comparatively higher proportion of metal over silicates in the inner part of the solar nebula than further away from the Sun. They include the contrasting condensation temperatures for different phases (metal vs. silicates) in the nebula, the dynamical phenomenon of metal and silicate separation that is photophoresis [the process whereby intense light, as from the Sun, can cause small particles in a very dilute medium to move and separate] (Wurm et al. 2013), and aerodynamic sorting due to the physical properties of silicate and metal bodies in the gaseous nebula (Weidenschilling 1978).

Volatility-dependent metal-silicate fractionation at medium temperatures $(1,300-1,700 \mathrm{~K})$ and low pressure during the condensation of the nebula may occur because of variations of temperature with heliocentric distance (FIG. 3). This process might be responsible for the metal enrichment of some meteorites and perhaps of Mercury, because $\mathrm{Fe}$ metal starts to condense at higher temperatures than $\mathrm{Mg}$ silicates at high total pressure (Ebel and Grossman 2000). Calculations of condensation sequences using vapors of solar composition show that a very high-density region (i.e., high-metal fraction but still including silicates) where Mercury may form directly by condensation occurs at a relatively high pressure of 0.01 to 0.001 bar in the nebula and at temperatures of 1,400-1,600 K. However, condensation temperatures of Fe-alloy and forsterite, which are the two major components of planetesimals, vary greatly from one model to another, thereby making it difficult to precisely estimate under what conditions Mercury, with its current metal/silicate ratio, may have formed directly by condensation.

Wurm et al. (2013) suggested that photophoresis could preferentially push silicate dust away from the Sun because of the low thermal conductivity of such dust compared to metal micronuggets. This would deplete the inner-most solar system of silicates. Aerodynamic sorting prior to accretion might also result in a metal-rich inner nebula because of the progressive decay in orbits with planetesimals as a result of gas drag (Weidenschilling 1978). This latter process could be responsible for the more effective removal of brittle, comminuted silicates compared to tougher iron during rotation of the inner nebula because orbital decay is slower for denser, larger bodies. To fully appreciate how these dynamic processes may have contributed to the formation of Mercury, additional information on the chemical, physical, and magnetic environment in which Mercury formed would be needed.

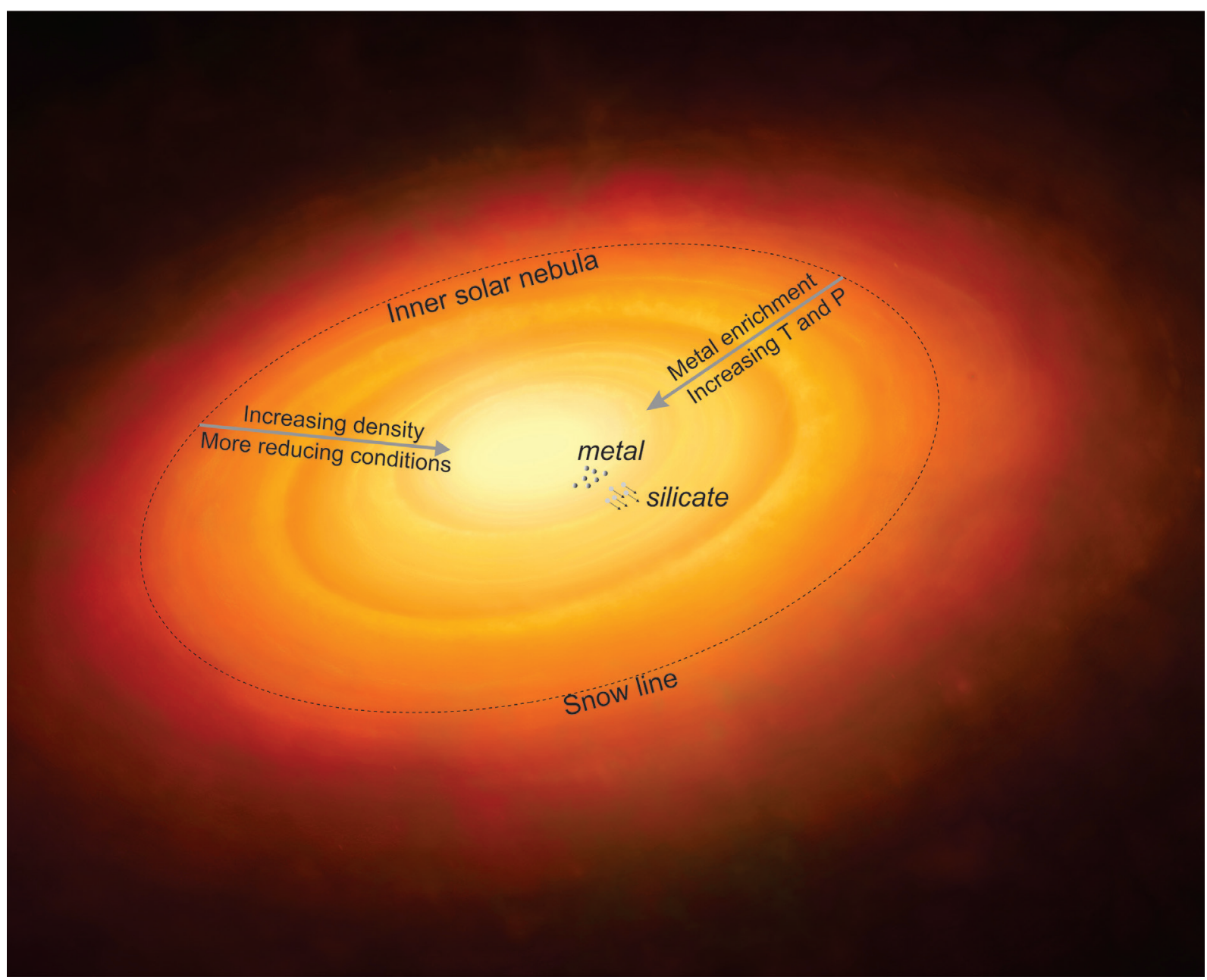

Figure 3

Key processes that affected the early inner sola nebula. Enrichment of metal over silicate materials in the inner solar nebula may be due to the higher condensation temperature for metal compared to silicate or to photophoretic separation of metal and silicate. Under this scenario, the high metal/silicate ratio of Mercury (and of other bodies from the inner solar system, such as enstatite chondrite meteorites) is inherited from primordial nebular processes. The snow line is the approximate distance from the Sun where volatiles condense into solid ice grains. Figure Copyright @ Mark A. Garlick. 


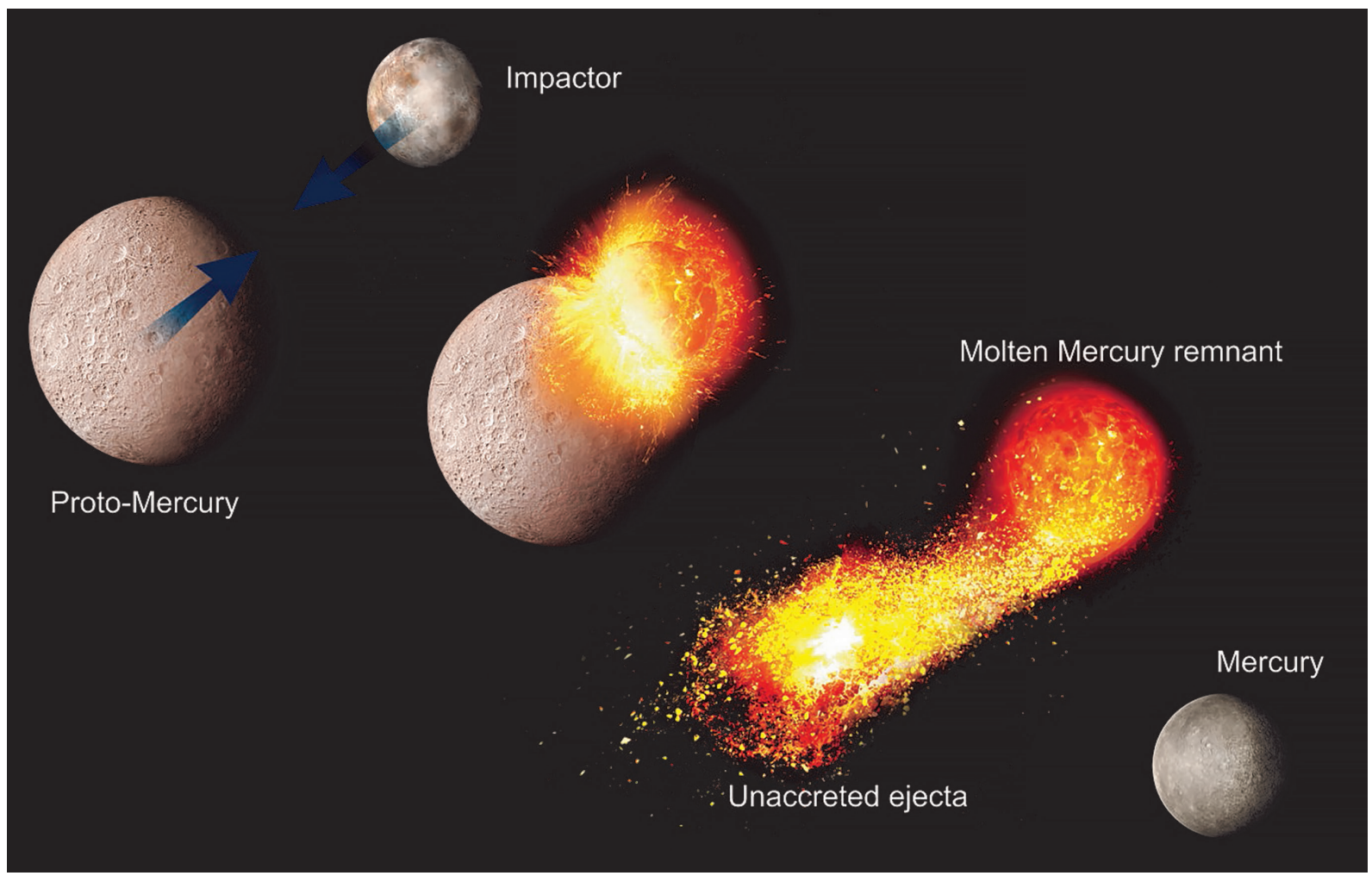

Figure 4 Early impact stripping of the silicate portion of Mercury. A giant collision between a relatively large proto-Mercury and a smaller planet could be responsible for the origin of the high metal/silicate ratio in Mercury today. This collision is responsible for the ejection of material from the whole outer silicate portion of the planet. Most of this material must not subsequently be reaccreted in order to leave a metal-rich core and a smaller silicate portion. Figure Copyright () Mark A. Garlick.

\section{Collisional Stripping and Inefficient Accretion}

The high metal/silicate ratio of Mercury could have been inherited during collisional processes through successive impacts that might have been responsible for rapid collisional stripping of the silicate portion of the planet (Benz et al. 1988) (FIG. 4). This process is also referred to as collisional or cratering erosion, and a range of such impactrelated scenarios has been considered (Asphaug and Reufer 2014). One or more head-on or "hit-and-run" collisions (with a range of possible impact parameters, such as the velocity and relative size and mass of target and impactor, and the impact angle) between proto-Mercury and another body or bodies would be responsible for the ejection of material from the outer silicate portion of the planet. This ejecta cloud would not subsequently reaccrete, meaning that such a collision would affected the whole volume of the planet to leave a metal-rich core and a smaller silicate outer portion.

\section{Post-Accretion Evaporation Model}

A molten proto-Mercury may have been exposed to very high temperatures, which could have been responsible for $70 \%-80 \%$ vaporization of the silicate portion of the planet (Fegley and Cameron 1987). Temperatures in the range $2,500-3,500 \mathrm{~K}$ may have been reached in the primitive inner part of the solar nebula for a significant period of time, vaporizing silicate material that could have been carried away by the solar winds. This process, however, is not appropriate to explain the origin of Mercury because this model would predict that the planet should be depleted in alkalis and enriched in titanium relative to chondrite material, but it is not. However, the model itself is of interest because a disintegrating super-Mercury (same density but larger than Mercury) has possibly been observed by NASA's Kepler space telescope around a dwarf star with macroscopic particles escaping its atmosphere (Rappaport et al. 2012). Although probably not applicable to Mercury, such a process could potentially explain the formation of some high-density exoplanets.

\section{PERSPECTIVES}

Mercury, the innermost planet of our solar system, might not be exceptional when one considers the range of rocky exoplanets. Some exoplanets are now recognized as composed of rock and iron and with mass-radius relationships that classify them as sub-Mercury-sized, Mercurylike, or super-Mercury planets (e.g., Barclay et al. 2013). In addition to the models mentioned above for the origin of Mercury, very dense iron planets have been suggested to potentially represent the remnant cores of gas giants (Mocquet et al. 2014). The burgeoning field of research on exoplanets will contribute to and benefit from a better understanding of Mercury. The ESA/JAXA BepiColombo spacecraft is now on its way to explore Mercury in even greater detail. A new era in the understanding of planet Mercury will start as soon as the first data from its two comprising spacecraft (the Mercury Planetary Orbiter and the Mercury Magnetospheric Orbiter) will be sent back to Earth. However, one of the ultimate future goals is to land on Mercury and collect in situ chemical and mineralogical measurements: this should be a priority of future missions. Until then, the collection of enstatite achondrites (aubrites) and unclassified meteorites should be further scrutinized as being potential "messages" from Mercury.

\section{ACKNOWLEDGMENTS}

We thank Mark A. Garlick for designing the figures; Jean Clair Duchesne and Jurrien Knibbe for valuable comments on the original manuscript; and reviewers Nancy Chabot, Anne Pommier and Denton Ebel for improving the final text. = 


\section{REFERENCES}

Asphaug E, Reufer A (2014) Mercury and other iron-rich planetary bodies as relics of inefficient accretion. Nature Geoscience 7: 564-568

Barclay T and 57 coauthors (2013) A sub-Mercury-sized exoplanet. Nature 494: 452-454

Benz W, Slattery WL, Cameron AGW (1988) Collisional stripping of Mercury's mantle. Icarus 74: 516-528

Blewett DT and 17 coauthors (2011) Hollows on Mercury: MESSENGER evidence for geologically recent volatilerelated activity. Science 333: 1856-1859

Byrne PK and 5 coauthors (2014) Mercury's global contraction much greater than earlier estimates. Nature Geoscience 7: 301-307

Cartier C, Wood BJ (2019) The role of reducing conditions in building Mercury. Elements 15: 39-45

Chabot NL, Wollack EA, Klima RL, Minitti ME (2014) Experimental constraints on Mercury's core composition. Earth and Planetary Science Letters 390: 199-208

Dumberry M, Rivoldini A (2015) Mercury's inner core size and corecrystallization regime. Icarus 248 : 254-268

Ebel DS, Grossman L (2000)

Condensation in dust-enriched systems. Geochimica et Cosmochimica Acta 64: 339-366

Ebel DS, Stewart ST (2018) The elusive origin of Mercury. In: Solomon SC, Anderson BJ, Nittler LR (eds) Mercury: The View after MESSENGER. Cambridge University Press, Cambridge, pp 497-515

Fegley B Jr, Cameron AGW (1987) A vaporization model for iron/silicate fractionation in the Mercury protoplanet. Earth and Planetary Science Letters 82: 207-222
Hauck SA II and 12 coauthors (2013) The curious case of Mercury's internal structure. Journal of Geophysical Research: Planets 118: 1204-1220

Knibbe JS, van Westrenen W (2018) The thermal evolution of Mercury's $\mathrm{Fe}-\mathrm{Si}$ core. Earth and Planetary Science Letters 482: 147-159

Li Y, Dasgupta R, Tsuno K (2017) Carbon contents in reduced basalts at graphite saturation: implications for the degassing of Mars, Mercury, and the Moon. Journal of Geophysical Research: Planets 122: 1300-1320

Malavergne V, Toplis MJ, Berthet S, Jones J (2010) Highly reducing conditions during core formation on Mercury: implications for internal structure and the origin of a magnetic field. Icarus 206: 199-209

Marchi S and 5 coauthors (2013) Global resurfacing of Mercury 4.0-4.1 billion years ago by heavy bombardment and volcanism. Nature 499: 59-61

Mocquet A, Grasset O, Sotin C (2014) Very high-density planets: a possible remnant of gas giants. Philosophical Transactions of the Royal Society A: Mathematical, Physical and Engineering Sciences 372, doi: 10.1098/ rsta.2013.0164

Namur O, Charlier B (2017) Silicate mineralogy at the surface of Mercury. Nature Geoscience 10: 9-13

Namur O, Charlier B, Holtz F, Cartier C, McCammon C (2016a) Sulfur solubility in reduced mafic silicate melts: implications for the speciation and distribution of sulfur on Mercury. Earth and Planetary Science Letters 448: 102-114

Namur O and 5 coauthors (2016b) Melting processes and mantle sources of lavas on Mercury. Earth and Planetary Science Letters 439: 117-128
Nittler LR, Weider SZ (2019) The surface composition of Mercury. Elements 15: $33-38$

Padovan S, Wieczorek MA, Margot J-L, Tosi N, Solomon SC (2015) Thickness of the crust of Mercury from geoid-totopography ratios. Geophysical Research Letters 42: 1029-1038

Peplowski PN and 10 coauthors (2014) Enhanced sodium abundance in Mercury's north polar region revealed by the MESSENGER Gamma-Ray Spectrometer. Icarus 228: 86-95

Peplowski PN and 9 coauthors (2016) Remote sensing evidence for an ancient carbon-bearing crust on Mercury. Nature Geoscience 9: 273-276

Rappaport S and 10 coauthors (2012) Possible disintegrating short-period super-Mercury orbiting KIC 12557548.

The Astrophysical Journal 752, doi: 10.1088/00004-637X/752/1/1

Solomon SC, Byrne PK (2019) The exploration of Mercury by spacecraft. Elements 15: 15-20

Thomas RJ, Rothery DA (2019) Volcanism on Mercury. Elements 15: 27-32

Vander Kaaden KE, McCubbin FM (2015) Exotic crust formation on Mercury: consequences of a shallow, FeO-poor mantle. Journal of Geophysical Research: Planets 120: 195-209

Weidenschilling SJ (1978) Iron/silicate fractionation and the origin of Mercury. Icarus 35: 99-111

Wurm G, Trieloff M, Rauer H (2013) Photophoretic separation of metals and silicates: the formation of Mercurylike planets and metal depletion in chondrites. The Astrophysical Journal 769, doi: 10.1088/0004-637X/769/1/78

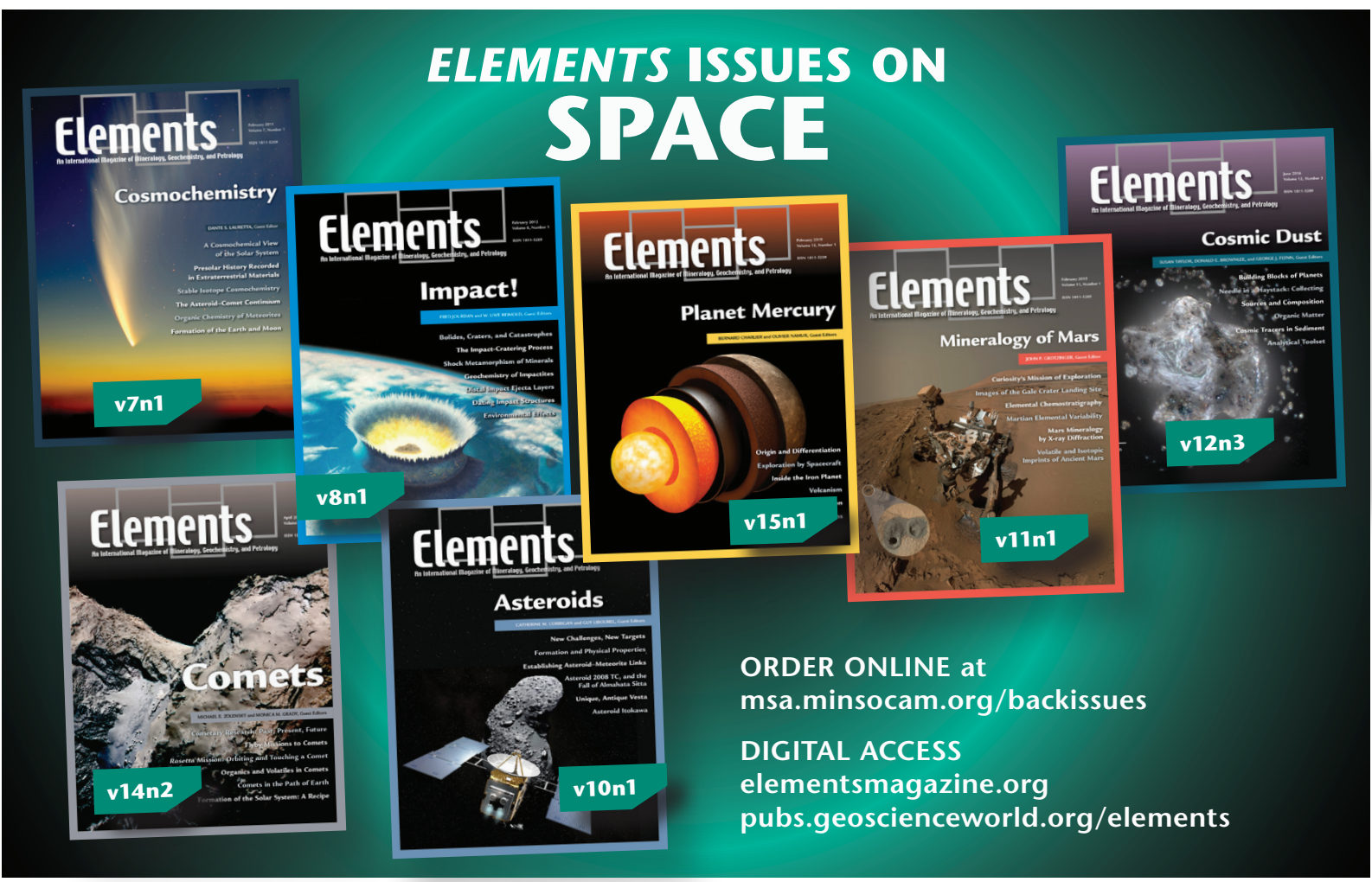

